\title{
Shock cardiogénico por infarto agudo del miocardio manejado con hemofiltración de alto volumen: Caso clínico
}

\author{
Daniel da Costaㄹ, Luigi G abrielli2, Max Andresen ${ }^{3}$, \\ Patricio Downey ${ }^{4}$, Pablo Castro². \\ Cardiogenic shock secondary to \\ acute myocardial infarction managed \\ with high volume hemofiltration: \\ Report of a case
}

Cardiogenic shock secondary to acute myocardial infarction unveils a systemic inflammatory response with elevation of cytokines that contribute to hypoperfusion. High volume hemofiltration may remove cytokines in patients with septic shock resulting in hemodynamic improvement and reducing the requirements of norepinephrine. We report a 48 year-old male with cardiogenic shock secondary to acute myocardial infarction who presented a systemic inflammatory response characterized by fever and hemodynamic collapse, without evidence of infection. Its hemodynamic profile was stabilized with high volume hemofiltration (Rev Méd Chile 2009; 137: 1357-62).

(Key words: Hemofiltration; Myocardial infarction; Shock, cardiogenic)

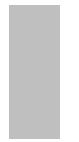

Recibido el 2 de diciembre, 2008. Aceptado el 7 de julio, 2009.

${ }^{1}$ Residente de Medicina Interna, ${ }^{2}$ Departamento de Enfermedades Cardiovasculares, ${ }^{3}$ Departamento de Medicina Intensiva, ${ }^{4}$ Departamento de Nefrología, Facultad de Medicina, Pontificia Universidad Católica de Chile. Santiago de Chile.

$E^{1}$ shock cardiogénico es un estado de hipoperfusión tisular debido a una severa falla cardíaca. Generalmente es debido a un infarto agudo al miocardio (IAM), siendo esta la causa más fre-

$\overline{\text { Correspondencia a: Daniel da Costa Gidi. Marcoleta } 3676^{\circ} \text { piso, }}$ Santiago, Chile. Fono: 09-4251850. E mail: danieldacosta@vtr,net Pablo Castro Gálvez. Fono/Fax: 02-6392037. E mail: pcastro@med.puc.cl cuente de muerte en los pacientes hospitalizados con IAM $^{1}$. Se define por el descenso del gasto cardíaco que produce vasoconstricción compensatoria, con evidencia de hipoxia tisular en presencia de un adecuado volumen intravascular. Sus criterios hemodinámicos son: hipotensión arterial (presión sistólica $<90 \mathrm{mmHg}$ por al menos 30 $\mathrm{min}$ ), índice cardíaco (IC) reducido $(<2,2 \mathrm{~L} / \mathrm{min}$ por $\mathrm{m}^{2}$ ) y presión capilar pulmonar (PCP) elevada $(>15 \mathrm{mmHg})^{2}$.

Este modelo fisiopatológico del shock cardiogénico ha ido evolucionando principalmente gra- 
cias al estudio SHOCK, protocolo aleatorizado de revascularización precoz en shock cardiogénico por $\mathrm{IAM}^{3-5}$. Sus resultados mostraron que la fracción de eyección (FE) sólo estuvo moderadamente deprimida (promedio de 30\%), y no existió vasoconstricción compensatoria, ya que la resistencia vascular sistémica (RVS) con vasopresores fue en promedio 1.350 a 1.400 dinas $\cdot \mathrm{s} \cdot \mathrm{cm}^{-5}$ (Tabla 1). Por otro lado, cabe destacar que 18\% de los pacientes presentó un síndrome de respuesta inflamatoria sistémica (SIRS), evidenciada por fiebre (94\%), leucocitosis (72\%) y RVS menores que el promedio, en los cuales se consideró el diagnóstico de sepsis o shock mixto. Este grupo presentó mayor mortalidad. De estos pacientes, 26\% tuvieron todos sus cultivos microbiológicos negativos, atribuyéndose el cuadro a un SIRS por el IAM ${ }^{4}$. Esta evidencia ha permitido sugerir que en determinados individuos el shock cardiogénico se debe a una disfunción cardíaca asociada a un SIRS que produce vasodilatación inapropiada y mayor depresión miocárdica, que en conjunto conducen al shock ${ }^{5-7}$.

Este SIRS ha sido explicado por una importante elevación de citoquinas $^{8-11}$, que inducen un aumento de la expresión génica de óxido nítrico sintetasa inducible $e^{5,12}$. Es así como se ha demostrado liberación miocárdica de interleuquina 6 (IL6) en $\mathrm{IAM}^{13}$, que puede llegar a niveles de la misma magnitud que los pacientes con shock séptico $^{14}$, siendo esta un factor independiente de mortalidad $^{11}$. Además, recientemente se ha repor- tado que aquellos pacientes con IAM y shock cardiogénico presentan niveles de IL-6 y factor de necrosis tumoral alfa (TNF- $\alpha$ ) mayores que aquellos con IAM sin shock cardiogénico ${ }^{8}$. Por otra parte, se han relacionado los niveles de IL-1ß y TNF- $\alpha$ con depresión miocárdica en pacientes sépticos ${ }^{15}$.

En pacientes con shock séptico grave ha sido empleada con éxito la hemofiltración de alto volumen (HFAV), mejorando fundamentalmente aspectos macrohemodinámicos ${ }^{16}$. Esta intervención ha demostrado una tendencia a disminuir la mortalidad del shock séptico, tanto hiperdinámi$\mathrm{co}^{17}$, como hipodinámico ${ }^{16}$. Es utilizada preferentemente en el shock séptico refractario, logrando disminuir en forma significativa la dosis de noradrenalina en aproximadamente la mitad de los pacientes, postulándose que esto lo logra por la remoción de citoquinas ${ }^{17}$. Su uso en otros tipos de shock con hiperrespuesta inflamatoria, como algunos casos de shock cardiogénico por IAM, no ha sido reportado.

Se presenta un caso de shock cardiogénico por IAM reperfundido, que presentó una falla circulatoria catastrófica con shock refractario, sin evidencia de infección, manejado exitosamente con HFAV.

\section{Caso Clínico}

Hombre de 48 años, con antecedente familiar de cardiopatía coronaria, tabáquico y dislipidémico sin

Tabla 1. M ediciones hemodinámicas en el estudio SH OCK

\begin{tabular}{|lccc|}
\hline & SIRS & & Control no-SIRS \\
& & 18\% & \\
& Cultivos & & \\
& positivos 13,5\% & & $\begin{array}{c}\text { Cultivos } \\
\text { negativos 4,7\% }\end{array}$ \\
\hline FE (\%) & $30 \pm 12$ & $25 \pm 9$ & $28 \pm 11$ \\
RVS (dinas $\left.\cdot \mathrm{s} \cdot \mathrm{cm}^{-5}\right)$ & 1.051 & 1.174 & 1.402 \\
& $(862-1.486)$ & $(705-1.370)$ & $(1.088-1.807)$ \\
\hline
\end{tabular}

Datos obtenidos al inicio del shock cardiogénico.

SIRS: síndrome de respuesta inflamatoria sistémica (definido por fiebre y leucocitosis). FE: fracción de eyección. RVS: resistencia vascular sistémica. 
tratamiento farmacológico. Presentó intenso dolor precordial mientras jugaba fútbol, consultando en un servicio de urgencia donde presentó paro cardiorrespiratorio por fibrilación ventricular. Fue tratado con múltiples desfribilaciones y maniobras de reanimación durante $25 \mathrm{~min}$. El electrocardiograma (ECG) mostró supradesnivel del segmento ST en la pared anterior. Se trasladó al Hospital Clínico de la Pontificia Universidad Católica de Chile para estudio hemodinámico de urgencia, con IAM de 9 h de evolución. Al ingreso se encontraba intubado con asistencia ventilatoria mediante ambú, hemodinámicamente inestable requiriendo infusión de noradrenalina a $0,1 \mu \mathrm{g} / \mathrm{kg} / \mathrm{min}$. El ECG mostró onda Q y supradesnivel del segmento ST hasta $3 \mathrm{~mm}$ en V1 a V3. Troponina I: $292 \mathrm{ng} / \mathrm{mL}$ (normal $<0,05$ ).

Se realizó coronariografía a las $10 \mathrm{~h}$ de iniciado el IAM, que mostró oclusión total de la arteria descendente anterior (ADA) proximal, con coronaria derecha y circunfleja sin lesiones, efectuándose angioplastia con balón en ADA, con flujo final TIMI II. Se realizó ecocardiograma que evidenció akinesia no adelgazada antero-septoapical, determinando una severa disfunción sistólica con FE estimada en $25 \%$, sin otro hallazgo patológico.

Se trasladó a Unidad Coronaria donde presentó mayor compromiso hemodinámico, requiriendo noradrenalina a $0,6 \mu \mathrm{g} / \mathrm{kg} / \mathrm{min}$, oligoanuria e hiperlactacidemia de $5,6 \mathrm{mmol} / \mathrm{L}$ (normal $<2,4$ ). Debido a su mala evolución se realizó nuevo estudio coronariográfico a las $22 \mathrm{~h}$ de iniciado el IAM, que mostró flujo TIMI 3 en ADA, sin lesión residual significativa. Se decidió apoyo ventricular con balón de contrapulsación intraaórtico y asociar adrenalina como vasopresor. Su perfil hemodinámico mostró: presión arterial de 94/63 $\mathrm{mmHg}$, IC $5,3 \mathrm{~L} / \mathrm{min} / \mathrm{m}^{2}$, PCP $23 \mathrm{mmHg}$ y RVS 784 dinas $\cdot \mathrm{s} \cdot \mathrm{cm}^{-5} / \mathrm{m}^{2}$.

Su temperatura central era de $38,5^{\circ} \mathrm{C}$, con leucocitosis de $25.400 / \mathrm{mm}^{3}$ y proteína $\mathrm{C}$ reactiva de $23,7 \mathrm{mg} / \mathrm{dL}$ (normal $<1$ ). Se realizaron cultivos e imágenes buscando algún foco infeccioso, que incluyeron radiografía de tórax y escáner de tórax, abdomen y pelvis, todo lo cual resultó negativo para infección, atribuyéndose el shock distributivo secundario a IAM extenso no reperfundido en forma precoz.
Debido a lo refractario del compromiso hemodinámico, a pesar de un elevado aporte de drogas vasoactivas y balón de contrapulsación intraaórtico, se decidió tratar en forma empírica con HFAV con el objetivo teórico de remover citoquinas inflamatorias, tal como lo reportado para el manejo del shock séptico refractario ${ }^{17}$. Se inició HFAV a $80 \mathrm{~mL} / \mathrm{kg} / \mathrm{h}$ durante $6 \mathrm{~h}$, manteniendo un balance neutro mediante la reposición de una solución con la siguiente composición: sodio $140 \mathrm{mEq} / \mathrm{L}$, potasio $2 \mathrm{mEq} / \mathrm{L}$, calcio $3 \mathrm{mEq} / \mathrm{L}$, magnesio $1 \mathrm{mEq} / \mathrm{L}$, dextrosa $100 \mathrm{mg} / \mathrm{dL}$ y osmolalidad 296 $\mathrm{mOsm} / \mathrm{L}$. Se utilizó un filtro de polisulfona altamente biocompatible de $1,4 \mathrm{~m}^{2}$, con un volumen extracorpóreo de $243 \mathrm{~mL}$. En asociación con la HFAV se observó disminución de drogas vasoactivas en $30 \%$, aumento de la RVS a 949 dinas $\cdot \mathrm{s} \cdot \mathrm{cm}^{-5} / \mathrm{m}^{2}$ y reducción del IC a $2,7 \mathrm{~L} / \mathrm{min} / \mathrm{m}^{2}$. La temperatura del paciente durante la terapia fue de $37,9^{\circ} \mathrm{C}$ promedio. Al día siguiente, recibe segunda HFAV durante $6 \mathrm{~h}$, lográndose suspender la adrenalina (Tabla 2).

Se dio de alta a los 48 días con un cardiodesfibrilador implantado a las 6 semanas de iniciado el cuadro, como profilaxis primaria debido a la mala función ventricular residual.

\section{DisCUSIÓN}

Nuestro paciente tuvo un IAM extenso de pared anterior del ventrículo izquierdo, como consecuencia de oclusión aguda aterotrombótica de ADA reperfundido con angioplastia percutánea con balón a las 10 h de evolución del IAM, y que presentó shock cardiogénico asistido mediante balón de contrapulsación intraaórtico, complicado por un SIRS caracterizado por fiebre, leucocitosis y falla hemodinámica que requirió de altas dosis de noradrenalina y adrenalina, sin evidencia de infección.

Este comportamiento no puede ser explicado por la fisiopatología clásica del shock cardiogénico, donde sólo existe una depresión de la contractilidad miocárdica que provoca un síndrome de bajo gasto cardíaco, hipotensión arterial e insuficiencia coronaria. Tal como propuso Hochmam, este paradigma debe ser extendido gracias a los análisis del estudio SHOCK $^{5}$, agregándose la 
Tabla 2. Respuesta hemodinámica en el paciente a la hemofiltración de alto volumen

\begin{tabular}{|lcccc|}
\hline $\begin{array}{l}\text { H oras desde } \\
\text { iniciado el IAM }\end{array}$ & Hora $\mathbf{3 4}$ & Hora $\mathbf{4 0}$ & H ora $\mathbf{5 0}$ & H ora $\mathbf{5 6}$ \\
\hline Situación clínica & & & & Término de \\
& Inicio de & Término de & Inicio de & segunda HFAV \\
Vasoactivos & primera HFAV & primera HFAV & segunda HFAV & NA: 0,12 \\
& NA: 0,71 & NA: 0,50 & NA: 0,23 & A: 0 \\
Presión arterial $(\mathrm{mmHg})$ & $94 / 63$ & A: 0,021 & A: 0,018 & $126 / 76$ \\
\hline
\end{tabular}

IAM: infarto agudo al miocardio. HFVA: hemofiltración de alto volumen. NA: noradrenalina en $\mu \mathrm{g} / \mathrm{kg} / \mathrm{min}$. A: adrenalina en $\mu \mathrm{g} / \mathrm{kg} / \mathrm{min}$.

presencia de un SIRS secundario a la liberación de citoquinas que produce una mayor depresión miocárdica y vasodilatación inapropiada, ambas mediadas en parte por óxido nítrico (Figura 1).

La presencia de falla hemodinámica refractaria a uso de drogas vasoactivas, en contexto de un miocardio ya reperfundido y asistido, con patrón cardiovascular distributivo hiperdinámico, nos hizo plantear que el SIRS dado por citoquinas estaba complicando al shock cardiogénico. Esto es compatible con los hallazgos del estudio de Lim et $\mathrm{al}^{7}$, en que algunos pacientes con shock cardiogénico murieron posterior a una rápida normalización de su IC, con un patrón de shock distributivo en la ausencia de infección, probablemente atribuido a citoquinas y óxido nítrico. Estos pacientes

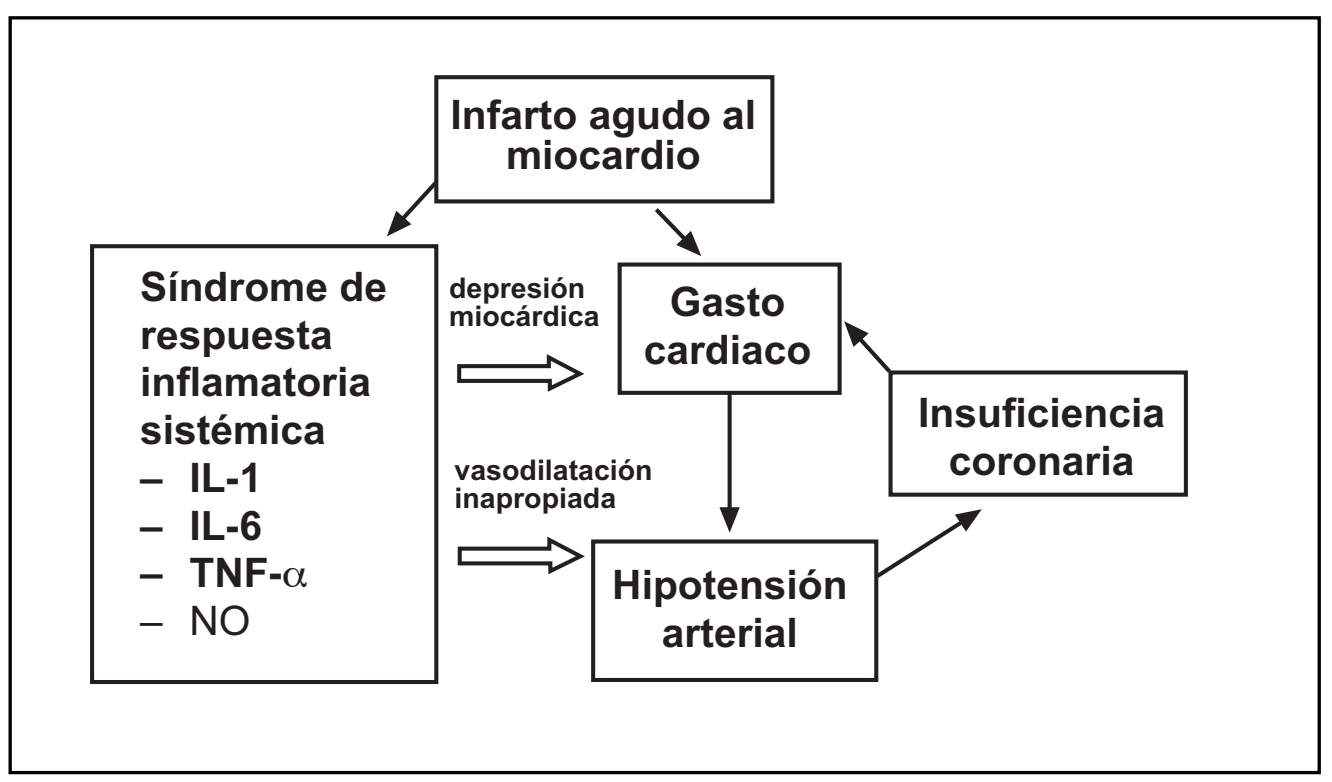

Figura 1. La influencia del síndrome de respuesta inflamatoria sistémica en la fisiopatología del shock cardiogénico. (IL: interleuquina, TNF- $\alpha$ : factor de necrosis tumoral alfa, NO: óxido nítrico) (esquema propio de los autores). 
eran más jóvenes y tenían una mayor reserva cardíaca, tal como nuestro paciente, pudiendo estos factores contribuir en un SIRS más pronunciado.

Extrapolando información existente del tratamiento del shock séptico con HFAV ${ }^{17}$, se decidió emplear esta técnica lográndose rápida y significativa mejoría hemodinámica. Probablemente los mecanismos involucrados en este caso son semejantes a los postulados en el shock séptico. En esta entidad se postula que la ultrafiltración y absorción por el hemofiltro es capaz de controlar niveles excesivamente elevados de citoquinas y otros factores inflamatorios. Esto produce un equilibrio entre agentes pro y antiinflamatorios, lo que estabiliza la hemodinamia.

La hemofiltración se ha empleado en pacientes con insuficiencia cardíaca refractaria a terapia convencional como medida depurativa ${ }^{18}$, sin em-

\section{REFERENCIAS}

1. Hochman JS, Boland J, Sleeper la, Porway M, Brinker J, Col J ET AL. Current Spectrum of Cardiogenic Shock and Effect of Early Revascularization on Mortality. Results of an International Registry. SHOCK. Registry Investigators. Circulation 1995; 91: 873-81.

2. Forrester JS, Diamond G, Chayyerjee K, Swan HJ. Medical Therapy of Acute Myocardial Infarction by Application of Hemodynamic Subsets. N Engl J Med 1976; 295: 1404-13.

3. Hochman J, Sleeper L, Webb J, Sanborn T, White H, Talley D ET aL. Early Revascularization in Acute Myocardial Infarction Complicated by Cardiogenic Shock. N Engl J Med 1999; 341, 9: 625-34.

4. Kohsaka S, Menon V, Lowe A, Lange M, Dzavik V, SLEEPER L ET AL. Systemic Inflammatory Response Syndrome After Acute Myocardial Infarction Complicated by Cardiogenic Shock. Arch Intern Med 2005; 165: 1643-50.

5. Hochman JS. Cardiogenic shock complicating acute myocardial infarction: expanding the paradigm. Circulation 2003; 107: 2998-3002.

6. Reynolds H, Hochman J. Cardiogenic Shock Current Concepts and Improving Outcomes. Circulation 2008; 117: 686-97.

7. Lim N, Dubois M, Backer D, Vincent J. Do All bargo, publicaciones acerca de HFAV como terapia de rescate en shock cardiogénico por IAM con falla circulatoria y vasodilatación inapropiada no han sido reportados.

En conclusión, en pacientes con shock cardiogénico por IAM con patrón hemodinámico distributivo la HFAV puede constituir una herramienta terapéutica para el manejo hemodinámico. Los elementos beneficiosos podrían ser atribuidos a la remoción de citoquinas. Se requieren estudios con mayor número de pacientes y medición de niveles de citoquinas para confirmar estos hallazgos.

\section{Agradecimientos}

Los autores agradecen a los doctores Alberto Dougnac y Marcelo Mercado por su contribución en la explicación de los mecanismos fisopatológicos involucrados en el caso clínico.
Nonsurvivors of Cardiogenic Shock Die With a Low Cardiac Index? Chest 2003; 124: 1885-91.

8. Debrunner M, Schuiki E, Minder E, Straumann E, Naegeli B, Mury R et al. Proinflammatory cytokines in acute infarction with and without cardiogenic shock. Clin Res Cardiol 2008; 97: 298-305.

9. Lindmark E, Diderholm E, Wallentin L, Siegbahn A. Relationship between interleukin 6 and mortality in patients with unstable coronary artery disease: Effects of an early invasive or noninvasive strategy. JAMA 2001; 286: 2107-13.

10. Yip H, Youssef A, Chang L, Yang C, Sheu J, Chua S et AL. Association of Interleukin-10 Level With Increased 30-Day Mortality in Patients With ST-Segment Elevation Acute Myocardial Infarction Undergoing Primary Coronary Intervention. Circ J 2007; 71: 1086-91.

11. Geppert A, Dorninger A, Delle-Karth G, Zorn G, Heinz G, Huber K. Plasma concentration of interleukin-6, organ failure, vasopressor support, and successful coronary revascularization in predicting 30-day mortality of patients with cardiogenic shock complicating acute myocardial infarction. Crit Care Med 2006; 34, 8: 2036-42.

12. Akiyama K, Kimura A, Suzuki H, Takeyama Y, Gluckman T, Terhakopian A et al. Production of oxidative products of nitric oxide in infarcted human heart. J Am Coll Cardiol 1998; 32: 373-9. 
13. Neumann FJ, Ott I, Gawaz M, Richardt G, Holzapfel H, Jochum M ET AL. Cardiac release of cytokines and inflammatory responses in acute myocardial infarction. Circulation 1995; 92: 748-55.

14. Geppert A, Steiner A, Zorn G, Delle-Karth G, Koreny M, Haumer M et al. Multiple organ failure in patients with cardiogenic shock is associated with high plasma levels of interleukin-6. Crit Care Med 2002; 30: 1987-94.

15. Kumar A, Thota V, Dee L, Olson J, Uretz E, Parrillo J. Tumor necrosis factor $\mathrm{a}$ and interleukin $1 \beta$ are responsible for in vitro myocardial cell depression induced by human septic shock serum. J Exp Med 1996; 183: 949-58.
16. Honore P, Jamez J, Wauthier M, Lee P, Dugernier T, Pirenne B ET Al. Prospective evaluation of short-term, high-volume isovolemic hemofiltration on the hemodynamic course and outcome in patients with intractable circulatory failure resulting from septic shock. Crit Care Med 2000; 28: 3581-7.

17. Cornejo R, Downey P, Castro R, Romero C, Regueira T, VEGA J ET AL. High-volume hemofiltration as salvage therapy in severe hyperdynamic septic shock. Int Care Med 2006; 42: 713-22.

18. Inoue T, Sakai Y, Morooka S, Takayanagi K, Hayashi T, TABajaKe Y. Hemofiltration as treatment for patients with refractory heart failure. Clin Cardiol 1992; 15: 514-8. 